\title{
Implementation of community case management of malaria in malaria endemic counties of western Kenya: are community health volunteers up to the task in diagnosing malaria?
}

Enock Oburi Marita ( $\square$ enockmarita@gmail.com )

Amref Health Africa https://orcid.org/0000-0001-7923-1271

Bernard Langat

Amref Health Africa

Teresa Kinyari

University of Nairobi

Patrick Igunza

Amref Health Africa

Donald Apat

Amref Health Africa

Josephat Kimori

Amref Health Africa

Jane Carter

Amref Health Africa

Richard Kiplimo

Kenya Ministry of Health

Samuel Muhula

Amref Health Africa

Research

Keywords: Community case management of malaria, community health volunteers, test positivity rate, Kappa score, mRDTs

Posted Date: August 30th, 2021

DOl: https://doi.org/10.21203/rs.3.rs-845559/v1

License: (9) (1) This work is licensed under a Creative Commons Attribution 4.0 International License. Read Full License 
Version of Record: A version of this preprint was published at Malaria Journal on March 5th, 2022. See the published version at https://doi.org/10.1186/s12936-022-04094-w. 


\section{Abstract}

\section{Background}

Community case management of malaria $(\mathrm{CCMm})$ is an equity-focused strategy that complements and extends the reach of health services by providing timely and effective management of malaria to populations with limited access to facility-based healthcare. In Kenya, CCMm involves the use of malaria rapid diagnostic tests (mRDT) and treatment of confirmed uncomplicated malaria cases with artemether lumefantrine (AL) by community health volunteers (CHVs). The test positivity rate (TPR) from CCMm reports collected by the Ministry of Health in 2018 was twofold compared to facility-based reports for the same period. This necessitated the need to evaluate the performance of $\mathrm{CHVs}$ in conducting malaria RDTs.

Method

The study was conducted in four counties within the malaria lake endemic zone in Kenya with a malaria prevalence in 2018 of $27 \%$; the national prevalence of malaria was $8 \%$. Multi-stage cluster sampling and random selection were used. Results from 200 malaria RDTs performed by CHVs were compared with test results obtained by experienced medical laboratory technicians (MLT) performing the same test under the same conditions. Blood slides prepared by the MLTs were examined microscopically later as a backup check of the results. A kappa score was calculated to assess level of agreement. Sensitivity, specificity, positive and negative predictive values were calculated to determine diagnostic accuracy.

Results

The median age of CHVs was 46 (IQR: 38,52$)$ with a range $[26,73]$ years. Females were $72 \%$ of the CHVs. Test positivity rates for MLTs was $42 \%$ and for CHVs was $41 \%$. The kappa score was 0.89 indicating an almost perfect agreement in mRDT results between CHVs and MLTs. The overall sensitivity and specificity between the CHVs and MLTs were $95.0 \%$ (95\% Cl: $(87.7,98.6)$ and $94.0 \%(95 \% \mathrm{Cl} ; 88.0,97.5)$ respectively.

\section{Conclusion}

Engaging $\mathrm{CHVs}$ to diagnose malaria cases under $\mathrm{CCMm}$ yielded results which compared well with results of qualified experienced laboratory personnel. CHVs can reliably continue to offer malaria diagnosis in the community setting.

\section{Background}

Early detection and treatment of malaria contributes to reduced complications and deaths (1). A mixed approach of providing health services at both health facility and community levels is appropriate where only about $70 \%$ of people in Sub-Saharan Africa use public facilities as the first point of care when a family member has fever (1). 
Globally, given the limited human resources in the health sector, the community-based approach has been promoted as a cost-effective and pro-poor intervention to improve the accessibility of healthcare (2), (3) and (1). This underscores the importance of community health volunteers ( $\mathrm{CHVs}$ ) as a key element in the community-based approach to most populations in low- and middle-income countries (4). CHVs are generally defined as non-professional lay health workers who work in the communities where they reside, and who are equipped with training and incentives to provide promotional, preventive and curative healthcare services to community members (5), Bhattacharyya et al., 2001 and (6)

In Kenya, CHVs are recruited at community meetings (barazas) called by area leaders or community health committees (CHC), using set criteria. (7). CHVs are organised into community units (CUs) supervised by community health extension workers (CHEWs). Each $\mathrm{CHV}$ provides services to an average of 100 households, linking them to the formal health sector; about 5,000 community members are served by CHVs within each community unit (Kimberly, Kristen, \& Tanvi, 2017); (8).

Community case management of malaria $(\mathrm{CCMm})$ is an equity-focused strategy that complements and extends the reach of health services by providing timely and effective diagnosis and treatment to populations with limited access to facility-based healthcare (9). In Kenya, the CCMm strategy utilizes CHVs who have received training on performing and interpreting malaria rapid diagnostic tests (mRDT) and prescribe artemether lumefantrine $(\mathrm{AL})$ to confirmed, uncomplicated malaria cases. CHVs refer pregnant women with suspected malaria, suspected severe malaria cases, patients with negative malaria test results and patients with persistent symptoms to health facilities for further management.

In western Kenya, a malaria endemic zone, CCMm has been adapted increasingly since 2012 as an approach to increase timely access to malaria care and treatment. About 7,420 CHVs have been trained and equipped with health commodities and tools to promptly diagnose and treat uncomplicated malaria cases at community level and help prevent progression to severe life-threatening disease. CHVs are also trained to identify severe malaria cases for early referral and thus help reduce malaria deaths. CHVs are part of the first level of national malaria monitoring, and conduct epidemiological surveillance of malaria cases at community level. CHVs submit monthly reports to the Kenya Health Information System (KHIS) and thus contribute to the national malaria control strategy with up-to-date information (10).

Symptom-based malaria diagnosis is inaccurate and contributes to poor management of febrile illness, over-treatment of malaria, and may promote drug resistance to current anti-malarial drugs (11). The World Health Organization recommends testing of all suspected malaria cases before treatment as best practice in malaria case management (12). The 2019-2023 Kenya Malaria Strategy emphasises this recommendation with testing in healthcare facilities using microscopy and mRDT (13). While microscopy is the diagnostic test of choice in health facilities with laboratories, mRDTs are used in facilities where microscopy is unavailable due to several factors such as lack of microscopes, trained laboratory personnel or electricity. Testing of malaria in the community setting is entirely by mRDT with the intention of reducing the practice of presumptive malaria treatment and irrational use of malaria treatment drugs. 
While microscopy detects the presence of malaria parasites in blood by direct observation, mRDT detects the presence of circulating malaria parasite antigens. The most commonly used mRDT detects Plasmodium falciparum-specific histidine-rich protein 2 (PfHRP2) while others detect lactate dehydrogenase (LDH) and aldolase. mRDT results may remain positive for a variable amount of time (561 days) following effective treatment with anti-malarial drugs, depending on the type of mRDT used, age and treatment, thereby affecting their specificity (14). Sensitivity is associated with the inherent performance of the test, as well as quality issues related to handling of test kits and the performance of the testing procedure. Although $\mathrm{CHVs}$ undergo training on the use of $\mathrm{mRDT}$, storage and transport conditions and human error may affect the validity of the test results. Procedural factors include the quality of the blood drop as well as the time taken by the operator to read the test results (15)

The National Malaria Control Programme uses routine surveillance data reported in the Kenya Health Information System (KHIS) to produce a quarterly Malaria Surveillance Bulletin. In the July to September 2018 issue, the all-age malaria test positivity rate (TPR) was $24 \%$ with the TPR in the malaria lake endemic zone being comparatively high at $35 \%$ (16). However, these data are not disaggregated to facilities or community level. From the CCMm routine data reported for the same period, the average TPR for malaria RDTs performed by CHVs in the malaria lake endemic zone was almost twofold at $67 \%$. There was therefore a need to evaluate the performance of $\mathrm{CHVs}$ in conducting RDTs and determine the accuracy of their reports in comparison with tests performed by qualified laboratory personnel.

\section{Methods}

The study was conducted in Kakamega, Vihiga, Siaya and Migori Counties which are in the malaria lake endemic zone in Kenya, between September and October 2020. The study population was selected from $\mathrm{CHVs}$ conducting CCMm in these counties. The climate in this area is mainly tropical, with variations due to altitude, and rainfall all year round with warm temperatures that influence mosquito populations and malaria transmission. Main sources of livelihood are agriculture, small scale businesses and fishing. There are about $9000 \mathrm{CHV}$ s covering about $30 \%$ of the population and 385 public health facilities in the four counties (17).

This was a cross-sectional survey to evaluate the performance of $\mathrm{CHVs}$ in testing for malaria using mRDTs. A quantitative subset of the study data was used where $200 \mathrm{CHVs}$ were observed conducting mRDT on 200 patients. These results were then compared with results from mRDTs performed by experienced medical laboratory technicians (MLTs) using a second sample of capillary blood from the same patient. Blood films were also prepared and examined in the laboratory setting by WHO Level One microscopists as back up verification as required. In cases with discordant results, the mRDT results of the MLTs were used to manage patients.

Multi-stage cluster sampling was used to select the study sample with the sampling frame as the eight malaria endemic counties, sub-counties and community units (CUs) where CCMm is practised. The first stage involved random sampling of four counties based on their predominantly cultural backgrounds. 
The sample was then proportionately apportioned to the four counties based on the number of CUs implementing $\mathrm{CCMm}$. The second stage was a random selection of sub-counties from each randomly selected county, followed by a random selection of CUs from the selected sub-counties. Consecutive sampling was then used to identify five $\mathrm{CHVs}$ who had encountered a suspected malaria case from a sampled $\mathrm{CU}$, that is, from a sampled $\mathrm{CU}$ only $5 \mathrm{CHVs}$ were being observed.

Research assistants (RAs) were trained on study procedures before starting data collection. CHVs tested patients presenting with symptoms and signs of malaria; consenting patients with suspected uncomplicated malaria were included in the study. Patients with suspected severe or complicated malaria, pregnant women and children below one year were excluded. The RDT brand used was CareStart Malaria $^{\mathrm{TM}}$ (AccessBio, USA), obtained from the government central stores using the usual procedures; storage and handling strictly met the manufacturer's guidelines.

Medical laboratory technicians (MLT) independently performed an mRDT (same brand and batch number as used by the $\mathrm{CHV}$ ) on the same participant by performing a second prick to collect capillary blood. MLTs also prepared thick and thin blood films (on the same slide) for back-up microscopy. The CHVs and MLTs were blinded to the results of each other. Thin films were fixed in methanol and air-dried, then films were stained with $10 \%$ Giemsa solution for 15 minutes. Staining was done within 12 hours to avoid autofixation of films. Malaria parasites were recorded as the number of asexual parasites counted per 500 white blood cells in the thick film. If no parasite was found in at least 100 fields at 1,000 magnification in the thick film, the result was recorded as no malaria parasites seen. All thick and thin blood films were read by two WHO certified Level 1 microscopists and any discrepancy resolved by a third Level 1 microscopist. All microscopists were blinded to the results of the malaria RDTs.

For ease of tracking and analysis, the study participants were given unique identifier numbers containing the $\mathrm{CU}$ code, $\mathrm{CHV}$ code and study subject number. The MRDT strip and blood slides were labelled with the same unique identifier numbers. A log with a record of the mRDT results (from the CHV and MLTs) and blood slides were maintained using the identifier number; only the study coordinator had access to this log to maintain the blinding of test results.

Data were captured using electronic Open Data Kit (ODK). The log data kept by the research assistants (RA) were entered at the end of each day into ODK using a tablet. The data were transmitted to a server hosted by Amref Health Africa in real-time. At the end of each study day, data transmitted to the server were reviewed by the study coordinator and any quality issues flagged for immediate correction. The data were stored on a password protected computer with back-up on a password protected external hard-drive only accessible to authorised study staff. Quantitative data were downloaded from the server into Excel (Microsoft, USA) and transferred to STATA version 15 (Stata-Corp, College Station, Texas) for statistical analysis.

Cohen's kappa statistic for interrater reliability testing was calculated to establish the level of agreement between the $\mathrm{CHVs}$ and qualified laboratory staff. The kappa statistic (or kappa coefficient) was used to assess the strength of the agreement. Interpretation of kappa was as follows: $<0.20$ slight agreement, 
0.21-0.40 fair agreement, $0.41-0.60$ moderate agreement, $0.61-0.80$-substantial agreement, and 0.81-0.99-almost perfect agreement. Inter-reader agreement for facilities versus reference values was expressed as kappa ( $\mathrm{K}$ ) with 95\% confidence interval (Cl) using the 'kapci' function in Stata (18)

Sensitivity and specificity were calculated as the proportion of mRDT positive and negative test results obtained by the CHVs against the results of the MLTs. Positive and negative predictive values were calculated as the proportion of true positive results among all positive samples and the proportion of true negative results among all negative samples, respectively.

\section{Results}

A total of $200 \mathrm{CHV}$ participants were enrolled into the study, distributed proportionate to the size of each county, with $42 \%$ from Kakamega, 27\% from Migori, 18.5\% from Siaya and 12.5\% from Vihiga Counties. The socio-demographic characteristics of the study participants are shown in Table 1. The CHVs' median age was 45 (IQR: $43-47$ ) years, age range $26-75$ years. 
Table 1

Socio-demographic characteristics of the study participants

\begin{tabular}{|c|c|c|}
\hline Characteristic & Category & Overall \\
\hline \multirow[t]{2}{*}{ Sex } & Male & $57(28.5 \%)$ \\
\hline & Female & $143(71.5 \%)$ \\
\hline \multirow{5}{*}{$\begin{array}{l}\text { Age group } \\
\text { (years) }\end{array}$} & $26-35$ & $36(18 \%)$ \\
\hline & $36-45$ & $64(32 \%)$ \\
\hline & $46-55$ & $67(33 \%)$ \\
\hline & $56-65$ & $29(14 \%)$ \\
\hline & $66+$ & $4(2 \%)$ \\
\hline \multirow[t]{5}{*}{ Years of experience } & $<1$ & $14(7 \%)$ \\
\hline & $1-2$ & $76(38 \%)$ \\
\hline & $3-5$ & $43(21 \%)$ \\
\hline & $>5$ & $49(24 \%)$ \\
\hline & Missing & $18(9 \%)$ \\
\hline \multirow[t]{3}{*}{ Marital status } & Single & $3(1.5 \%)$ \\
\hline & Married & $174(87 \%)$ \\
\hline & Widowed & $23(11.5 \%)$ \\
\hline \multirow[t]{5}{*}{ Education Level } & Completed primary education & $47(23.5 \%)$ \\
\hline & Some primary education & $18(9 \%)$ \\
\hline & Completed secondary education & $62(31 \%)$ \\
\hline & Some secondary education & $63(31.5 \%)$ \\
\hline & College education & $10(5 \%)$ \\
\hline \multirow[t]{2}{*}{ Religion } & Christian & $194(97 \%)$ \\
\hline & Muslim & $6(3 \%)$ \\
\hline
\end{tabular}

At $95 \%$ confidence interval $(0.82,0.95)$, the kappa score was 0.89 indicating almost perfect agreement (92.5\%) in mRDT results between CHVs and MLTs. The standard error was 0.07 and Prob > Z was 0.000 .

The overall sensitivity and specificity between CHVs and MLTs were 95.0\% (95\% Cl: $(87.7,98.6)$ and $94.0 \%(95 \% \mathrm{Cl} ; 88.0,97.5)$ respectively (Table 2$)$. The sensitivity for male and female CHVs was $100 \%$ 
(95\% Cl: $(83.9,100)$ and $93.2 \%(95 \% \mathrm{Cl} ; 83.5,98.1)$ respectively, while the specificity was $97.1 \%$ (95\% Cl: $(84.7,99.9)$ and $92.7 \%(95 \% \mathrm{Cl} ; 84.8,97.3)$ respectively.

Table 2

Sensitivity, specificity, positive and negative predictive values of RDTs performed by CHVs compared to MLTs, segregated by gender

\begin{tabular}{|llll|}
\hline CHV vs MLT & & & \\
\hline \multirow{2}{*}{ Overall } & Characteristic & PE & $(95 \% \mathrm{Cl})$ \\
\hline & Sensitivity & 95.0 & $(87.7,98.6)$ \\
\hline & Specificity & 94.0 & $(88.0,97.5)$ \\
\hline Male & Positive predictive value & 91.6 & $(83.4,96.5)$ \\
\hline & Negative predictive value & 96.5 & $(91.2,99)$ \\
\hline Female & Sensitivity & 100.0 & $(83.9,100)$ \\
\hline & Specificity & 97.1 & $(84.7,99.9)$ \\
\hline & Positive predictive value & 95.5 & $(77.2,99.9)$ \\
\hline & Negative predictive value & 100.0 & $(89.4,100)$ \\
\hline SE = Point estimate; CHV = Interpretation by Community Health & Volunteer; MLT = Interpretation by & \\
\hline Medical Laboratory Technician & 93.2 & $(83.5,98.1)$ \\
\hline
\end{tabular}

Overall sensitivity and specificity between CHVs performing RDTs and microscopy were $91.1 \%$ (95\% Cl: $(82.6,96.4)$ and $88.8 \%(95 \% \mathrm{Cl} ; 81.6,93.9)$ respectively (Table 3). Comparable performance in sensitivity and specificity were $91.6 \%$ (95\% Cl: $(83.4,96.5)$ and $91.2 \%$ (95\% Cl; 84.5, 95.7) respectively between MLTs performing RDTs and microscopy. 
Table 3

Sensitivity, specificity, positive predictive value and negative predictive value, segregated by gender

\begin{tabular}{|c|c|c|c|c|c|}
\hline \multicolumn{2}{|c|}{ Characteristic } & \multicolumn{4}{|c|}{ Malaria cases (Pos vs Neg) } \\
\hline & & \multicolumn{2}{|c|}{ CHV vs Microscopy } & \multicolumn{2}{|c|}{ MLT vs Microscopy } \\
\hline & & PE \% & $(95 \% \mathrm{Cl})$ & PE \% & $(95 \% \mathrm{Cl})$ \\
\hline \multirow[t]{4}{*}{ Overall } & Sensitivity & 91.1 & $(82.6,96.4)$ & 91.6 & $(83.4,96.5)$ \\
\hline & Specificity & 88.8 & $(81.6,93.9)$ & 91.2 & $(84.5,95.7)$ \\
\hline & Positive predictive value & 84.7 & $(75.3,91.6)$ & 88.4 & $(79.7,94.3)$ \\
\hline & Negative predictive value & 93.6 & $(87.3,97.4)$ & 93.7 & $(87.4,97.4)$ \\
\hline \multirow[t]{4}{*}{ Male } & Sensitivity & 85 & $(62.1,96.8)$ & 86.4 & $(65.1,97.1)$ \\
\hline & Specificity & 91.2 & $(76.3,98.1)$ & 94.1 & $(80.3,99.3)$ \\
\hline & Positive predictive value & 85 & $(62.1,96.8)$ & 90.5 & $(69.6,98.8)$ \\
\hline & Negative predictive value & 91.2 & $(76.3,98.1)$ & 91.4 & $(76.9,98.2)$ \\
\hline \multirow[t]{4}{*}{ Female } & Sensitivity & 93.2 & $(83.5,98.1)$ & 93.4 & $(84.1,98.2)$ \\
\hline & Specificity & 87.8 & $(78.7,94)$ & 90 & $(81.2,95.6)$ \\
\hline & Positive predictive value & 84.6 & $(73.5,92.4)$ & 87.7 & $(77.2,94.5)$ \\
\hline & Negative predictive value & 94.7 & $(87.1,98.5)$ & 94.7 & $(87.1,98.5)$ \\
\hline
\end{tabular}

Comparing performance of diagnosing malaria by male and female CHVs with the MLTs, the sensitivity and specificity were $91.1 \%$ (95\% Cl: $(85,96.8)$ and $91.2 \%(95 \% \mathrm{Cl}: 76.3,98.1)$ for males respectively, and the sensitivity and specificity were $93.2 \%$ (95\% Cl: $(83.5,98.1)$ and $87.8 \%(95 \% \mathrm{Cl}: 78.7,94)$ for females respectively.

\section{Discussion}

Many endemic areas of the world lack sufficient capacity and resources to accurately diagnose malaria, and reliance on clinical signs and symptoms alone are inadequate and imprecise indicators of malaria disease. Use of $\mathrm{CHVs}$ has been shown to improve the acceptance of community interventions to address malaria diagnosis and treatment as $\mathrm{CHVs}$ are well respected within their communities of residence (19). The age and sex characteristics of the participating CHVs were similar to those in a study by (20) which evaluated mRDT use by community health workers. 
This study demonstrated consistently similar results between mRDTs conducted by CHVs and MLTs, and both results compared well with microscopy. A study done by (21), to compare RDTs and microscopy to diagnose malaria found $64 \%$ and $59 \%$ positivity of RDTs and microscopy respectively. The difference could be because RDTs detect malaria antigens and hence detect antigens still in circulation after recovery from the disease, giving false positive results, as compared to microscopy that detects parasite forms.

Results of kappa scores, sensitivity and specificity of this study were consistent with those of(20) which demonstrated that $\mathrm{CHVs}$ generally adhered to testing procedures, could safely and accurately perform RDTs, and interpreted test results correctly. While the findings contributed to the body of evidence that CHVs perform RDTs at an acceptable level (22), their skills were observed to improve with increasing years of experience. These findings could be as a result of routine support supervision and a good understanding of CCMm leading to its effective implementation.

Interrater reliability is important as it represents the extent to which the collected data in the study correctly represented the variables measured. The kappa statistic was used to test interrater reliability between the CHVs and MLTs. As guided by (18), the study kappa score was above $80 \%$ agreement which is the minimum acceptable interrater agreement, and an indication for an almost perfect agreement between CHVs and MLTs.

CHVs achieved very good sensitivity, specificity, positive predictive values and negative predictive values. These results are similar to those reported in most studies although they used microscopy as the gold standard for diagnosis (22).

The difference in malaria positivity rates as reported in the KHIS needs to be studied further to understand the root cause of the difference. The national malaria surveillance data may need to be disaggregated by $\mathrm{CHVs}$ and health facilities at different levels, with follow up of the different sources of data for verification.

\section{Conclusion}

The ability of $\mathrm{CHVs}$ to diagnose malaria cases under the CCMm project compared well with the findings of qualified, experienced laboratory staff as evidenced by comparable sensitivity, specificity, PPV, NPV and kappa scores. CCMm should continue and scale up its valuable and important role of first line diagnosis and treatment of uncomplicated malaria. Alternative possible causes of differing malaria positivity rates between those from $\mathrm{CHVs}$ and general national data needs to be explored.

\section{Declarations}

\section{Ethics approval and consent to participate}


Since human subjects were involved, ethics approval was obtained from the Amref Ethics and Scientific Review Committee with reference number P742-2019. There were minimum potential risks involved in this study. The malaria tests involved two finger pricks for drawing blood samples, one by the CHV and one by the laboratory technicians. Slight pain associated with sample collection was therefore anticipated. The study participants were expressly informed of this and consent for involvement in the study and a second sample collection sought. Written informed consent was obtained from all participants aged 18 years and above. Written assent was obtained from children aged 14 to 17 years together with written parental or guardian consent. Paper assent and consent forms were stored in a secure place only accessible to authorised study staff, and copies were provided to the study participants.

Privacy and confidentiality were observed and participation was voluntary. Participants were free to refuse to participate in the study or withdraw at any time without affecting the quality of the services they received from $\mathrm{CHVs}$.

\section{Consent for publication}

Not Applicable

\section{Availability of data and materials}

The datasets used and/or analysed during the current study are available from the corresponding author on reasonable request.

\section{Competing interests}

The authors declare that they have no competing interests.

\section{Funding}

Funding was from Global Fund to Fight AIDS, Tuberculosis and Malaria (GFATM). The funder did not play a role in the design of the study, or in collection, analysis and interpretation of the data, and writing the manuscript.

\section{Authors' contributions}

$\mathrm{BL}$ was the principal investigator coordinating and leading the research, $\mathrm{JC}$ was technical advisor and main reviewer of the manuscript, OA contributed to the study design and review of the manuscript, TK contributed to conceptual initiatives and design of the study, PI was key in the design of the study and data collection and analysis, JK supported data collection and writing of the manuscript, RK was the statistician supporting data analysis, interpretation of data and writing of the manuscript, SM was involved in data analysis and writing of the manuscript, EM was the lead/corresponding author, designed the study and led the collection, analysis and interpretation of the data and writing the manuscript. All authors read and approved the final manuscript 


\section{Acknowledgements}

We are grateful to the study respondents and the research team, and to the county governments of Migori, Vihiga, Kakamega and Siaya Counties.

\section{Authors' information (optional)}

Not applicable

\section{References}

1. WHO. World Malaria Report. 2019.

2. Improving Maternal and Newborn Health: Effectiveness of a Community Health Worker Program in Rural Kenya.. Adam, MB, et al. 2014, PLoS ONE., pp. 104027-8.

3. The Ghana Community-based Health Planning and Services Initiative for scaling up service delivery innovation. Health Policy Plann. Nyonator, FK, et al. 2005, PubMed, pp. 25-34.

4. Lehmann, Uta and Sanders, David. Community Health Workers: What Do We Know About Them? The State of the Evidence on Programmes, Activities, Costs and Impact on Health Outcomes of Using Community Health Workers. Evidence and Information. 2007.

5. Community health workers in low-and middle-income countries: what do we know about scaling up and sustainability? Pallas, Wood, Sarah, et al. 2013, PMC, pp. 74-82.

6. Global Experience of Community Health Workers for Delivery of Health Related Millennium Development Goals: A Systematic Review, Country Case Studies, and Recommendations for Integration into National Health Systems. Bhutta, Zulfiqar, et al. 2010.

7. Kimberly, Farnham, Egan, Kristen, Devlin and Tanvi, Pandit-Rajani. Community Health Systems Catalog Country Profile: Kenya. Arlington, VA: Advancing Partners \& Communities. Arlington: JSI RESEARCH \& TRAINING INSTITUTE, 2017.

8. $\mathrm{MOH}$. Reversing the trends: the second national health sector strategic plan: NHSSP II-2005-2010. Nairobi. Nairobi: MOH, 2005.

9. WHO. Integrated community case management of malaria. Geneva: World Health Organization, 2016.

10. MSH. https://www.pmi.gov/. US President's Malria Initiative. [Online] 2017. https://www.pmi.gov/news/stories-from-the-field/.

11. Malaria misdiagnosis: effects on the poor and vulnerable. Amexo, Mark, et al. 2004, PMC, pp. 18968.

12. WHO. Guidelines for the treatment of malaria. Geneva, Switzerland: World Health Organization, 2015.

13. NMCP. Kenya Malaria Strategy 2019-2023. Nairobi: National Malria Control Program, Ministry of Health, Kenya, 2019. 
14. Dalrymple, Ursula, et al. How long do rapid diagnostic tests remain positive after anti-malarial treatment? s.I.: BMC, 2018.

15. Update on Rapid Diagnostic Testing for Malaria. Murray, K. Clinton, et al. 2008, PMC, pp. 97-110.

16. NMCP. Malaria Surveillance bulletin. Nairobi, Kenya.: National Malaria Control Program, Kenya,MOH, Kenya, 2018.

17. MOH. Government of Kenya Ministry of Health, 2015, County Health fact sheets. County Health Fact Sheets. Nairobi: Ministry of Health, 2005.

18. Interrater reliability: the kappa statistic. McHugh, Mary L. 2012, Blochemia Medica, pp. 276-282.

19. Community-based intermittent mass testing and treatment for malaria in an area of high transmission intensity, western Kenya: development of study site infrastructure and lessons learned. Odero, N.A., Samuels, A.M. and Odongo, W. 2019, Malar J, p. 255.

20. Evaluation of malaria rapid diagnostic test (RDT) use by community health workers: a longitudinal study in western Kenya. Boyce, M.R., Menya, D. and Turner, E.L. 2018, Malar J, p. 206.

21. A comparative laboratory diagnosis of malaria: microscopy versus rapid diagnostic test kits.. Azikiwe, C.C, et al. 2012, Asian Pacific journal of tropical biomedicine., pp. 307 - 10.

22. Rapid diagnostic tests for diagnosing uncomplicated $P$. falciparum malaria in endemic countries. Abba, Katharine, et al. 2011, Cochrane. 